\title{
ON THE ABSTRACT PROPERTIES OF MARKOV GRAPHS FOR MAPS ON TREES
}

\author{
SERGIY KOZERENKO
}

\begin{abstract}
Having a dynamical system on the vertex set of a finite tree, one can construct the corresponding Markov graph which is the digraph that encodes covering relation between edges in a tree. Representatives of isomorphism classes of Markov graphs are called M-graphs. In this paper we prove that the class of M-graphs is closed under several prescribed digraph transformations (such as deletion of a vertex in a digraph or taking the disjoint union of digraphs, for example). We also give a complete list of tournaments which are M-graphs as well as of M-graphs with three vertices.
\end{abstract}

\section{INTRODUCTION}

A dynamical system is a pair $(X, f)$, where $X$ is some set and $f: X \rightarrow X$ is a map from $X$ to itself. Combinatorial dynamics mainly deals with the structure of periodic points and their orbits. If $X$ is a topological space and $f$ is a continuous map, then we obtain a topological dynamical system. The most natural example of such a system is provided by the closed unit interval $X=[0,1]$ and its continuous self-map $f:[0,1] \rightarrow[0,1]$. Sharkovsky's theorem [9] (which was proven in 1964) completely describes the coexistence of periods of periodic points for continuous maps on the interval. In $[2,11]$ (and also in many other papers) the authors presented a combinatorial proof of Sharkovsky's theorem which uses the so-called periodic graphs. Namely, if $x \in[0,1]$ is a periodic point of period $n \geq 2$ for the map $f:[0,1] \rightarrow[0,1]$, then the restriction of $f$ to the orbit $\sigma=\left.f\right|_{\text {orb }(x)}$ is a cyclic permutation of $\operatorname{orb}(x)$ (here $\left.\operatorname{orb}(x)=\left\{x, f(x), \ldots, f^{n-1}(x)\right\}\right)$. Now let $\operatorname{orb}(x)=\left\{x_{1}<x_{2}<\right.$ $\left.\cdots<x_{n}\right\}$ be the natural ordering of $\operatorname{orb}(x)$. We can encode the dynamics of minimal intervals $\left[x_{i}, x_{i+1}\right], 1 \leq i \leq n-1$ by the dynamics of their ends $x_{i}, x_{i+1}$. The corresponding periodic graph $\Gamma$ is a digraph with the vertex set $\{1, \ldots, n-1\}$ (each $1 \leq i \leq n-1$ represents the minimal interval $\left.\left[x_{i}, x_{i+1}\right]\right)$ and there is an arc $i \rightarrow j$ if $\min \left\{f\left(x_{i}\right), f\left(x_{i+1}\right)\right\} \leq x_{j}<\max \left\{f\left(x_{i}\right), f\left(x_{i+1}\right)\right\}$ (i.e. if $\left[x_{i}, x_{i+1}\right] f$-covers $\left.\left[x_{j}, x_{j+1}\right]\right)$. It is easy to see that each directed cycle

2010 Mathematics Subject Classification. Primary: 05C20, Secondary: 05C05, 37E25.

Key words and phrases. dynamics on trees, Markov graphs, tournaments. 
$C$ in $\Gamma$ corresponds to some periodic point of $f$ and if $C$ does not consists of a smaller cycle traced several times, then the period of this point equals to the length of $C$. Also, using this graph-theoretic approach one can prove an analogue of Sharkovsky's theorem for continuous maps on topological trees of a general form (see [1]).

The first graph-theoretic results concerning periodic graphs were obtained by Pavlenko $[6,7,8]$. In particular, in [6] the number of non-isomorphic periodic graphs with a given number of vertices was counted. Criteria for an arbitrary digraph to be a periodic graph or to be an induced subgraph of a periodic graph were presented in [7] and [8], respectively.

In this paper we study graph-theoretical properties of Markov graphs of self-maps on finite trees. These are the natural generalization of periodic graphs.

\section{Definitions AND PRELIMINARY RESUlts}

This paper deals with undirected graphs without loops or multiple edges as well as with digraphs (which may have loops). All graphs and digraphs assumed to be finite. Thus, a graph $X$ is a pair $(V, E)$, where $V=V(X)$ is a set of vertices and $E=E(X)$ is a set of edges, which are just unordered pairs of vertices. The edge between two vertices $u$ and $v$ in a graph $X$ will be denoted simply as $u v \in E(X)$. The graph $X_{1}$ is a subgraph of a graph $X_{2}$ (denoted as $\left.X_{1} \subset X_{2}\right)$ if $V\left(X_{1}\right) \subset V\left(X_{2}\right)$ and $E\left(X_{1}\right) \subset E\left(X_{2}\right)$. For each set of vertices $A \subset V(X)$ in a graph $X$ put $E(A)=\{u v \in E(X): u, v \in A\}$. An induced subgraph $X[A]$ is a graph with $X[A]=(A, E(A))$. Similarly, for each set of edges $E^{\prime} \subset E(X)$ in a graph $X$ put $V\left(E^{\prime}\right)=\{u \in V(X)$ : $u$ is incident to some edge $\left.e \in E^{\prime}\right\}$. Thus, an induced subgraph $X\left[E^{\prime}\right]$ is a graph with $X\left[E^{\prime}\right]=\left(V\left(E^{\prime}\right), E^{\prime}\right)$. Also, given the sets $A \subset V(X)$ and $E^{\prime} \subset E(X)$ denote $X-A=X[V(X)-A]$ and $X-E^{\prime}=\left(V(X), E(X)-E^{\prime}\right)$.

For every vertex $u \in V(X)$ the set $N_{X}(u)=\{v \in V(X): u v \in E(X)\}$ is called its neighborhood. The number $d_{X}(u)=\left|N_{X}(u)\right|$ is called the degree of $u$. Also, put $\Delta(X)=\max \left\{d_{X}(u): u \in V(X)\right\}$. The vertex $u$ is called isolated provided $d_{X}(u)=0$. Similarly, the vertex $u$ is a leaf if $d_{X}(u)=1$. The set of all leaf vertices in a graph $X$ is denoted by $L(X)$. A leaf edge is an edge incident to some leaf vertex. A non-leaf edge is called inner.

A graph is called connected if there is a path between each pair of vertices. The vertex set $V(X)$ of a connected graph $X$ is equipped with the "shortest paths" metric $d_{X}(u, v), u, v \in V(X)$. For every vertex $u \in V(X)$ in a connected graph $X$ put $\operatorname{ecc}_{X}(u)=\max \left\{d_{X}(u, v): v \in V(X)\right\}$ for its eccentricity. Also, put $\operatorname{rad} X=\min \left\{\operatorname{ecc}_{X}(u): u \in V(X)\right\}$ and $\operatorname{diam} X=$ $\max \left\{\operatorname{ecc}_{X}(u): u \in V(X)\right\}$ for the radius and the diameter of a connected graph $X$. 
The metric interval between a pair of vertices $u, v \in V(X)$ in a connected graph $X$ is the set $[u, v]_{X}=\left\{x \in V(X): d_{X}(u, x)+d_{X}(x, v)=d_{X}(u, v)\right\}$. A set of vertices $A \subset V(X)$ is convex provided $[u, v]_{X} \subset A$ whenever $u, v \in$ $A$. Also, for each edge $u v \in E(X)$ in a connected graph $X$ define the set $A_{X}(u, v)=\left\{x \in V(X): d_{X}(x, u) \leq d_{X}(x, v)\right\}$.

A tree is a connected acyclic graph. Paths $P_{n}$ (i.e. $n$-vertex trees $X$ with $|L(X)| \leq 2)$ and stars $K_{1, n}$ (i.e. $(n+1)$-vertex trees $X$ with $\left.|L(X)|=n\right)$ are the most used examples of trees. Another class of trees that we will be using throughout this paper consists of spiders, i.e. trees with at most one vertex of degree at least three (thus, paths and stars are natural examples of spiders). If such a vertex $u$ exists, then we will call it as a center of a spider $X$ and its degree $d_{X}(u)$ as a degree of $X$. The paths $P_{1}, P_{2}$ and $P_{n}$ for $n \geq 3$ are spiders of degree zero, one and two, respectively.

A directed graph, or just a digraph $\Gamma$ is a pair $(V, A)$, where $V=V(\Gamma)$ is the set of vertices and $A=A(\Gamma) \subset V(\Gamma) \times V(\Gamma)$ is the set of arcs. There are some model digraphs. Namely, if $V(\Gamma)=\emptyset$, then $\Gamma$ is called trivial. If $A(\Gamma)=\emptyset$, then $\Gamma$ is called empty and denoted by $\bar{K}_{n}$ (here $n=|V(\Gamma)|$ ). If $A(\Gamma)=V(\Gamma) \times V(\Gamma)$, then $\Gamma$ is called complete and denoted by $K_{n}$ (here again, $n=|V(\Gamma)|)$.

For every digraph $\Gamma$ by $[\Gamma]$ we denote the undirected graph obtained from $\Gamma$ by "forgetting" the orientations of arcs. In other words, $V([\Gamma])=V(\Gamma)$ and $u v \in E([\Gamma])$ if $u \rightarrow v$ or $v \rightarrow u$ in $\Gamma$ for all $u, v \in V(\Gamma)$. If $[\Gamma]$ is connected, then $\Gamma$ is called weakly connected.

If there is an $\operatorname{arc}(u, v) \in A(\Gamma)$, then we will write $u \rightarrow v$ in $\Gamma$. Also, if there is no $\operatorname{arc}(u, v) \notin A(\Gamma)$, we will write sometimes just $u \nrightarrow v$ in $\Gamma$. The arc of the form $u \rightarrow u$ is called a loop at $u$. Also, put $N_{\Gamma}^{+}(u)=\{v \in V(\Gamma): u \rightarrow v\}$ and $N_{\Gamma}^{-}(u)=\{v \in V(\Gamma): v \rightarrow u\}$. The cardinalities $d_{\Gamma}^{+}(u)=\left|N_{\Gamma}^{+}(u)\right|$ and $d_{\Gamma}^{-}(u)=\left|N_{\Gamma}^{-}(u)\right|$ are called the outdegree and the indegree of $u$, respectively.

A directed cycle $\overrightarrow{C_{n}}$ with $n \geq 1$ vertices is a digraph of the form $u_{1} \rightarrow$ $\cdots \rightarrow u_{n} \rightarrow u_{1}$. For example, $\overrightarrow{C_{1}}=K_{1}$ is just a loop.

A tournament is an orientation of a complete graph. In other words, $\Gamma$ is a tournament if it does not contain cycles of length at most two and for each pair of vertices $u, v \in V(\Gamma)$ either $u \rightarrow v$ or $v \rightarrow u$ in $\Gamma$.

Consider two digraphs $\Gamma_{1}$ and $\Gamma_{2}$. By $\Gamma_{1} \cup \Gamma_{2}$ we denote their union, which is a digraph $\Gamma$ with $V(\Gamma)=V\left(\Gamma_{1}\right) \cup V\left(\Gamma_{2}\right)$ and $A(\Gamma)=A\left(\Gamma_{1}\right) \cup A\left(\Gamma_{2}\right)$. Similarly, by $\Gamma_{1} \sqcup \Gamma_{2}$ we denote their disjoint union, which is a digraph $\Gamma$ with $V(\Gamma)=V\left(\Gamma_{1}\right) \sqcup V\left(\Gamma_{2}\right)$ and $A(\Gamma)=A\left(\Gamma_{1}\right) \sqcup A\left(\Gamma_{2}\right)$. Further, by $\Gamma_{1} \rightarrow \Gamma_{2}$ we denote the digraph $\Gamma$ with $V(\Gamma)=V\left(\Gamma_{1}\right) \sqcup V\left(\Gamma_{2}\right)$ and $A(\Gamma)=A\left(\Gamma_{1}\right) \sqcup$ $A\left(\Gamma_{2}\right) \sqcup\left\{u \rightarrow v: u \in V\left(\Gamma_{1}\right), v \in V\left(\Gamma_{2}\right)\right\}$. Finally, we define the symmetric sum of $\Gamma_{1}$ and $\Gamma_{2}$ to be the digraph $\Gamma_{1}+\Gamma_{2}=\left(\Gamma_{1} \rightarrow \Gamma_{2}\right) \cup\left(\Gamma_{2} \rightarrow \Gamma_{1}\right)$. 
For every map of the form $f: X \rightarrow X$ one can consider its functional graph which is a digraph with the vertex set $X$ and there is an arc $x \rightarrow y$ if $f(x)=y$. Thus, the representatives of isomorphism classes of functional graphs are digraphs $\Gamma$ with $d_{\Gamma}^{+}(u)=1$ for all vertices $u \in V(\Gamma)$. Similarly, $\Gamma$ is called partial functional if $d_{\Gamma}^{+}(u) \leq 1$ for all vertices $u \in V(\Gamma)$.

In this paper we consider maps on vertex sets of trees. Let $X$ be a tree and $\sigma: V(X) \rightarrow V(X)$ be some map. The Markov graph $\Gamma=\Gamma(X, \sigma)$ is a digraph with the vertex set $V(\Gamma)=E(X)$ and there is an arc $e_{1} \rightarrow e_{2}$ if $u_{2}, v_{2} \in\left[\sigma\left(u_{1}\right), \sigma\left(v_{1}\right)\right]_{X}$, where $e_{i}=u_{i} v_{i}, i=1,2$. Thus, periodic graphs are Markov graphs $\Gamma(X, \sigma)$ for paths $X$ and their cyclic permutations $\sigma$. Representatives of isomorphism classes of Markov graphs are called M-graphs. In other words, $\Gamma$ is an M-graph if there exists a tree $X$ and its vertex map $\sigma: V(X) \rightarrow V(X)$ such that $\Gamma \simeq \Gamma(X, \sigma)$. For an M-graph $\Gamma$ each such a pair $(X, \sigma)$ will be called a realization of $\Gamma$. Also, in this case we will say that $\Gamma$ is realizable on $X$.

Example 1. Let $X$ be a tree with the vertex set $V(X)=\{1, \ldots, 5\}$ and the edge set $E(X)=\{12,23,34,25\}$. Consider the cyclic permutation $\sigma=$ (13524) of $V(X)$. Then the Markov graph $\Gamma(X, \sigma)$ is shown on Figure 1.

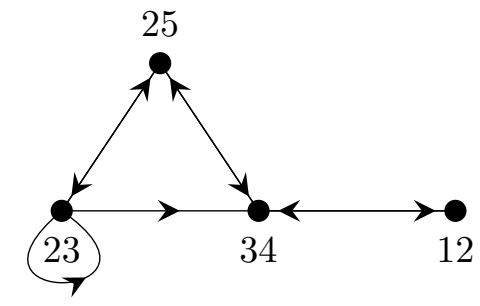

Figure 1. Markov graph $\Gamma(X, \sigma)$ for the pair $(X, \sigma)$ from Example 1.

We now state some basic properties of Markov graphs and maps on trees that we will use throughout this paper. Denote by $k(X)$ the number of connected components (i.e. maximal connected subgraphs) in $X$.

Proposition 1. [4] Let $X$ be a tree, $\sigma: V(X) \rightarrow V(X)$ be some map and $\Gamma=\Gamma(X, \sigma)$. For each edge $e=u v \in E(X)$ we have:

(1) $d_{\Gamma}^{+}(e)=d_{X}(\sigma(u), \sigma(v)) \leq \operatorname{diam} X$;

(2) $d_{\Gamma}^{-}(e)=k\left(X\left[\sigma^{-1}\left(A_{X}(u, v)\right)\right]\right)+k\left(X\left[\sigma^{-1}\left(A_{X}(v, u)\right)\right]\right)-1$.

Now let $X$ be a tree and $X^{\prime} \subset X$ be its subtree. It is easy to observe that for every vertex $u \in V(X)$ there exists a unique vertex $v \in V\left(X^{\prime}\right)$ such that $d_{X}(u, v)=\min \left\{d_{X}(u, x): x \in V\left(X^{\prime}\right)\right\}$ (in other words, the vertex set of each subtree is a Chebyshev set in the metric space $\left.\left(V(X), d_{X}\right)\right)$. Therefore, one can 
define a projection map $p r_{X^{\prime}}: V(X) \rightarrow V(X)$ putting $p r_{X^{\prime}}(u)=v$ for every $u \in V(X)$. It is easy to see that $p r_{e}^{-1}(u)=A_{X}(u, v)$ and $p r_{e}^{-1}(v)=A_{X}(v, u)$ for all edges $e=u v \in E(X)$.

Lemma 1. [3] Let $X$ be a tree, $\sigma: V(X) \rightarrow V(X)$ and $X^{\prime} \subset X$ be a subtree. Then $\Gamma\left[E\left(X^{\prime}\right)\right]=\Gamma\left(X^{\prime},\left.p r_{X^{\prime}} \circ \sigma\right|_{V\left(X^{\prime}\right)}\right)$.

For every tree $X$ and a map $\sigma: V(X) \rightarrow V(X)$ one can construct the edge labeling $\tau_{\sigma}: E(X) \rightarrow V(X) \cup\{1,-1\}$ in the following way. For each $e=u v \in E(X)$ we put $\tau_{\sigma}(e)=v$ if $\sigma(u), \sigma(v) \in A_{X}(v, u), \tau_{\sigma}(e)=1$ if $\sigma(u) \in A_{X}(u, v)$ and $\sigma(v) \in A_{X}(v, u), \tau_{\sigma}(e)=-1$ if $\sigma(u) \in A_{X}(v, u)$ and $\sigma(v) \in A_{X}(u, v)$. Thus, if $\tau_{\sigma}(e)=v$, then the edge $e=u v$ gets an orientation $u \rightarrow v$. Otherwise, the edge $e$ is $\sigma$-positive or $\sigma$-negative depending on the sign of $\tau_{\sigma}(e)$. The obtained mixed tree is a pair $\left(X, \tau_{\sigma}\right)$ which is denoted simply as $X\left(\tau_{\sigma}\right)$.

Example 2. Consider the pair $(X, \sigma)$ from Example 1. Then the mixed tree $X\left(\tau_{\sigma}\right)$ is shown on Figure 2 (the unique $\sigma$-negative edge is denoted by - ).

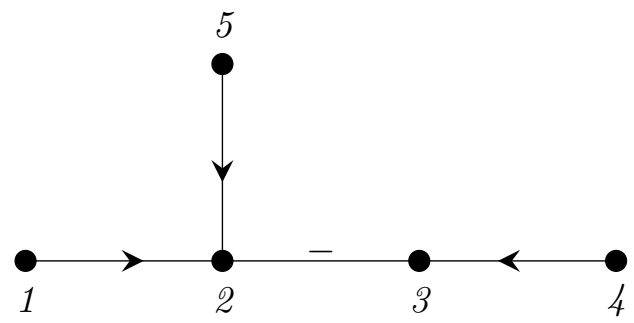

Figure 2. Mixed tree $X\left(\tau_{\sigma}\right)$ for the pair $(X, \sigma)$ from Example 1.

Denote by $p(X, \sigma)$ and $n(X, \sigma)$ the number of $\sigma$-positive and $\sigma$-negative edges in $X$, respectively. If $p(X, \sigma)=n(X, \sigma)=0$, then the map $\sigma$ is called anti-expansive. The map $\tau: E(X) \rightarrow V(X)$ is called an orientation of $X$ if $\tau(e)$ is incident to $e$ for all edges $e \in E(X)$.

Proposition 2. [5] Let $X$ be a tree and $\tau: E(X) \rightarrow V(X)$ be an orientation of edges in $X$. There exists an anti-expansive map $\sigma: V(X) \rightarrow V(X)$ with $\tau=\tau_{\sigma}$ if and only if there exists a vertex $u_{0} \in V(X)$ such that for every edge $e=u v \in E(X)$ from the equality $\tau(e)=v$ it follows that $v \in\left[u_{0}, u\right]_{X}$.

\section{TRANSFORMATIONS OF M-GRAPhS}

At first, observe that the empty digraph is an M-graph, since for every $n \geq 1$ and every tree $X$ with $n+1$ vertices each constant map $\sigma: V(X) \rightarrow$ $V(X)$ has an empty Markov graph $\Gamma(X, \sigma) \simeq \bar{K}_{n}$. Further, let $X$ be a path with $n+1$ vertices. For each of the two possible proper colorings $\sigma$ of $V(X)$ 
by the leaf vertices $L(X)$ the corresponding Markov graph $\Gamma(X, \sigma) \simeq K_{n}$ is a complete digraph. Therefore, every digraph is a subgraph of an M-graph. Moreover, as Pavlenko showed in [8] for any $n \geq 1$ there exists $m \geq 2 n-1$ and $m$-vertex periodic digraph which contain $K_{n}$ as a subgraph.

A digraph which is obtained from the complete digraph by the deletion of all loops will be called quasi-complete. Trivially, quasi-complete digraphs with at most two vertices are M-graphs. Meanwhile, the quasi-complete digraph with three vertices is not an M-graph, although it is an induced subgraph of an M-graph.

Example 3. Consider the tree $X$ from Example 1. Also, let $\sigma=\left(\begin{array}{lllll}1 & 2 & 3 & 4 & 5 \\ 5 & 4 & 1 & 5 & 1\end{array}\right)$.

Then the edges 12,25, 34 induce a quasi-complete digraph in $\Gamma(X, \sigma)$.

Proposition 3. Each quasi-complete digraph with at least four vertices is not an induced subgraph of an M-graph.

Proof. Consider a tree $X$, a map $\sigma: V(X) \rightarrow V(X)$ and suppose that the Markov graph $\Gamma=\Gamma(X, \sigma)$ contains an edge $e \in E(X)$ with $d_{\Gamma}^{+}(e) \geq 3$. Let $e_{1}, e_{2}, e_{3} \in N_{\Gamma}^{+}(e)$ be a triplet of distinct edges. Since all edges $e_{i}, 1 \leq i \leq 3$ lie on a common path in $X$, without loss of generality, we can assume that $e_{2}$ and $e_{3}$ lie in different connected components of $X-\left\{e_{1}\right\}$. Now, if $e_{2}, e_{3} \in N_{\Gamma}^{+}\left(e_{1}\right)$, then $e_{1} \in N_{\Gamma}^{+}\left(e_{1}\right)$. But this means that $e_{1}$ has a loop in $\Gamma$.

From Lemma 1 it strictly follows that each non-empty M-graph $\Gamma$ has a vertex $v \in V(\Gamma)$ such that $\Gamma-\{v\}$ is also an M-graph (the vertex $v$ corresponds to a leaf edge in a fixed realization $(X, \sigma)$ of $\Gamma)$. However, it is not true that for every vertex $v \in V(\Gamma)$ in an M-graph $\Gamma$ the digraph $\Gamma-\{v\}$ is also an M-graph. Consider the pair $(X, \sigma)$ from Example 3. Then $\Gamma(X, \sigma)-\{23\}$ is not an M-graph as it's the quasi-complete digraph with three vertices.

Proposition 4. Let $\Gamma$ be an $M$-graph and $v \in V(\Gamma)$ be its vertex with zero outdegree. Then $\Gamma-\{v\}$ is also an M-graph.

Proof. Fix an isomorphism $\Gamma \simeq \Gamma(X, \sigma)$. Let the edge $u_{1} u_{2} \in E(X)$ corresponds to the vertex $v$. Clearly, $\sigma\left(u_{1}\right)=\sigma\left(u_{2}\right)$. Contracting the edge $u_{1} u_{2}$ to a vertex, we obtain a new tree $X^{\prime}$ with $V\left(X^{\prime}\right)=\left(V(X)-\left\{u_{1}, u_{2}\right\}\right) \cup\left\{u^{\prime}\right\}$ (we assume that $\left.u^{\prime} \notin V(X)\right)$ and $E\left(X^{\prime}\right)=E\left(X-\left\{u_{1}, u_{2}\right\}\right) \cup\left\{u^{\prime} u: u \in\right.$ $\left.N_{X}\left(u_{1}\right) \cup N_{X}\left(u_{2}\right)\right\}$. Put

$$
\sigma^{\prime}(x)=\left\{\begin{array}{l}
\sigma(x), \text { if } x \neq u^{\prime} \text { and } \sigma(x) \notin\left\{u_{1}, u_{2}\right\}, \\
\sigma\left(u_{1}\right), \text { if } x=u^{\prime} \text { and } \sigma\left(u_{1}\right) \notin\left\{u_{1}, u_{2}\right\}, \\
u^{\prime}, \text { otherwise }
\end{array}\right.
$$

for all $x \in V\left(X^{\prime}\right)$. Then $\Gamma-\{v\} \simeq \Gamma\left(X^{\prime}, \sigma^{\prime}\right)$. 
Corollary 1. A digraph obtained from an M-graph by the deletion of an isolated vertex is also an M-graph.

Consider now two Markov graphs $\Gamma_{1}=\Gamma\left(X_{1}, \sigma_{1}\right)$ and $\Gamma_{2}=\Gamma\left(X_{2}, \sigma_{2}\right)$. Construct a new tree $X$ by adding the new edge between two fixed vertices: one from $X_{1}$ and the other one from $X_{2}$. Putting $\left.\sigma\right|_{V\left(X_{1}\right)}=\sigma_{1}$ and $\left.\sigma\right|_{V\left(X_{2}\right)}=$ $\sigma_{2}$ we obtain a Markov graph $\Gamma=\Gamma(X, \sigma)$. This means that for any pair of M-graphs $\Gamma_{1}$ and $\Gamma_{2}$ there exists an M-graph $\Gamma$ and its vertex $v \in V(\Gamma)$ such that $\Gamma-\{v\} \simeq \Gamma_{1} \sqcup \Gamma_{2}$. In particular, the disjoint union of two M-graphs is always an induced subgraph of an M-graph.

Recall that for a tree $X$ and a map $\sigma: V(X) \rightarrow V(X)$ the symbols $p(X, \sigma)$ and $n(X, \sigma)$ denote the number of $\sigma$-positive and $\sigma$-negative edges in $X\left(\tau_{\sigma}\right)$, respectively. Also, let fix $\sigma$ denotes the set of all fixed points of $\sigma$. In $[5]$ the following result was proved.

Theorem 1. [5] For every tree $X$ and a map $\sigma: V(X) \rightarrow V(X)$ we have

$$
n(X, \sigma)+\mid \text { fix } \sigma \mid=p(X, \sigma)+1 .
$$

As a corollary of Theorem 1, one can present a sufficient condition for the disjoint union of M-graphs to be an M-graph itself.

Proposition 5. Let $\Gamma_{1}$ and $\Gamma_{2}$ be two M-graphs with even numbers of loops in each. Then $\Gamma_{1} \sqcup \Gamma_{2}$ is an M-graph.

Proof. Fix isomorphisms $\Gamma_{i} \simeq \Gamma\left(X_{i}, \sigma_{i}\right), i=1,2$. From the definition it follows that the number of loops in $\Gamma_{i}, i=1,2$ equals $n\left(X_{i}, \sigma_{i}\right)+p\left(X_{i}, \sigma_{i}\right)$. Since this number is even, Theorem 1 asserts that $\sigma_{1}$ and $\sigma_{2}$ both have fixed points. Fix two of them $u_{1} \in f i x \sigma_{1}$ and $u_{2} \in f i x \sigma_{2}$. Consider the tree $X^{\prime}$ which is obtained by gluing $X_{1}$ and $X_{2}$ by the two vertices $u_{1}$ and $u_{2}$. In other words, let $V\left(X^{\prime}\right)=\left(V\left(X_{1}\right)-\left\{u_{1}\right\}\right) \cup\left(V\left(X_{2}\right)-\left\{u_{2}\right\}\right) \cup\left\{u^{\prime}\right\}$ (we assume that $V\left(X_{1}\right) \cap V\left(X_{2}\right)=\emptyset$ and $\left.u^{\prime} \notin\left(V\left(X_{1}\right)-\left\{u_{1}\right\}\right) \cup\left(V\left(X_{2}\right)-\left\{u_{2}\right\}\right)\right)$ and $E\left(X^{\prime}\right)=E\left(X_{1}-\left\{u_{1}\right\}\right) \cup E\left(X_{2}-\left\{u_{2}\right\}\right) \cup\left\{u^{\prime} u: u \in N_{X_{1}}\left(u_{1}\right) \cup N_{X_{2}}\left(u_{2}\right)\right\}$. Define the map $\sigma^{\prime}: V\left(X^{\prime}\right) \rightarrow V\left(X^{\prime}\right)$ in the following way:

$$
\sigma^{\prime}(x)=\left\{\begin{array}{l}
\sigma_{1}(x), \text { if } x \in V\left(X_{1}\right)-\left\{u_{1}\right\} \text { and } \sigma_{1}(x) \neq u_{1}, \\
\sigma_{2}(x), \text { if } x \in V\left(X_{2}\right)-\left\{u_{2}\right\} \text { and } \sigma_{2}(x) \neq u_{2}, \\
u^{\prime}, \text { otherwise }
\end{array}\right.
$$

for all $x \in V\left(X^{\prime}\right)$. Then $\Gamma_{1} \sqcup \Gamma_{2} \simeq \Gamma\left(X^{\prime}, \sigma^{\prime}\right)$.

Corollary 2. If $\Gamma_{1}$ and $\Gamma_{2}$ are two M-graphs without loops, then $\Gamma_{1} \sqcup \Gamma_{2}$ is also an M-graph.

Similarly, each digraph obtained from an M-graph by adding an isolated vertex is also an M-graph. To see this take an M-graph $\Gamma \simeq \Gamma(X, \sigma)$ and fix a leaf vertex $u \in L(X)$ in $X$. Then add a new vertex $u^{\prime}$ to $X$ with the new 
edge $u u^{\prime}$ obtaining a tree $X^{\prime}$. Putting $\sigma^{\prime}: V\left(X^{\prime}\right) \rightarrow V\left(X^{\prime}\right), \sigma^{\prime}\left(u^{\prime}\right)=\sigma(u)$ and $\sigma^{\prime}(x)=\sigma(x)$ for all $x \in V(X)$ we obtain $\Gamma \sqcup \bar{K}_{1} \simeq \Gamma\left(X^{\prime}, \sigma^{\prime}\right)$.

Remark 1. If $\Gamma_{1}$ and $\Gamma_{2}$ are two $M$-graphs such that there exist their realizations $\left(X_{i}, \sigma_{i}\right), i=1,2$ with fix $\sigma_{i} \neq \emptyset$, then $\Gamma_{1} \sqcup \Gamma_{2}$ is an M-graph. For example, the disjoint union of an M-graph with an even number of loops with a single loop $\vec{C}_{1}$ is also an M-graph. However, not every disjoint union of $M$-graphs is an M-graph. Consider $\Gamma$ as a digraph which is obtained from the complete digraph with two vertices by the deletion of one loop. Then $\Gamma$ is an M-graph, but $\Gamma \sqcup \vec{C}_{1}$ is not.

Now we introduce two local transformations of digraphs which are called doubling and reverse doubling of a vertex and then prove that the class of M-graphs is closed under these operations.

Consider some digraph $\Gamma$ and its vertex $v \in V(\Gamma)$. Add a new vertex $x$ to $\Gamma$ with new $\operatorname{arcs} x \rightarrow u$ for all vertices $u \in N_{\Gamma}^{+}(v)$ to obtain a new digraph $\Gamma^{\prime}$. We will say that $\Gamma^{\prime}$ is obtained from $\Gamma$ by the doubling of the vertex $v$.

Similarly, add a new vertex $y$ to $\Gamma$ with new $\operatorname{arcs} u \rightarrow y$ for all $u \in N_{\Gamma}^{-}(v)$ therefore obtaining a new digraph $\Gamma^{\prime}$. In this case, we will say that $\Gamma^{\prime}$ is obtained from $\Gamma$ by the reverse doubling of $v$.

Theorem 2. Let $\Gamma$ be an M-graph and let the digraph $\Gamma^{\prime}$ is obtained from $\Gamma$ by the doubling or the reverse doubling of some vertex. Then $\Gamma^{\prime}$ is an M-graph.

Proof. Fix an isomorphism $\Gamma \simeq \Gamma(X, \sigma)$. Suppose $\Gamma^{\prime}$ is obtained from $\Gamma$ by the doubling of the vertex $v \in V(\Gamma)$ and let $u_{1} u_{2} \in E(X)$ be the edge which corresponds to $v$. Add new leaf vertex $u$ to $X$ with the new edge $u u_{1}$ to obtain a new tree $X^{\prime}$ with $V\left(X^{\prime}\right)=V(X) \cup\{u\}$ and $E\left(X^{\prime}\right)=E(X) \cup\left\{u u_{1}\right\}$. Define $\sigma^{\prime}: V\left(X^{\prime}\right) \rightarrow V\left(X^{\prime}\right)$ in the following way:

$$
\sigma^{\prime}(x)=\left\{\begin{array}{l}
\sigma(x), \text { if } x \neq u, \\
\sigma\left(u_{2}\right), \text { if } x=u
\end{array}\right.
$$

for all $x \in V\left(X^{\prime}\right)$. It is easy to see that $\Gamma^{\prime} \simeq \Gamma\left(X^{\prime}, \sigma^{\prime}\right)$.

Now let $\Gamma^{\prime}$ is obtained from $\Gamma$ by the reverse doubling of the vertex $v \in V(\Gamma)$ and again let $u_{1} u_{2} \in E(X)$ be an edge corresponding to $v$. Subdivide the edge $u_{1} u_{2}$ by the new vertex $u$ to obtain a new tree $X^{\prime}$. In other words, let $V\left(X^{\prime}\right)=V(X) \cup\{u\}$ and $E\left(X^{\prime}\right)=\left(E(X)-\left\{u_{1} u_{2}\right\}\right) \cup\left\{u u_{1}, u u_{2}\right\}$. Define a map $\sigma^{\prime}: V\left(X^{\prime}\right) \rightarrow V\left(X^{\prime}\right)$ in the following way:

$$
\sigma^{\prime}(x)=\left\{\begin{array}{l}
\sigma(x), \text { if } x \neq u, \\
\sigma\left(u_{1}\right), \text { if } x=u
\end{array}\right.
$$

for all $x \in V\left(X^{\prime}\right)$. Again, we have $\Gamma^{\prime} \simeq \Gamma\left(X^{\prime}, \sigma^{\prime}\right)$. 
A map $\sigma: V\left(X_{1}\right) \rightarrow V\left(X_{2}\right)$ between vertex sets of two connected graphs $X_{1}$ and $X_{2}$ is called metric if $d_{X_{2}}(\sigma(u), \sigma(v)) \leq d_{X_{1}}(u, v)$ for all pairs of vertices $u, v \in V\left(X_{1}\right)$. It is easy to see that a map $\sigma$ is metric if and only if $d_{X_{2}}(\sigma(u), \sigma(v)) \leq 1$ for all edges $u v \in E\left(X_{1}\right)$. Combining this with Proposition 1, we can conclude that for a given tree $X$ the map $\sigma: V(X) \rightarrow V(X)$ is metric if and only if the Markov graph $\Gamma(X, \sigma)$ is partial functional.

Proposition 6. Let $\Gamma$ be a partial functional digraph. Then $\Gamma$ is an $M$-graph.

Proof. Let $\Gamma$ be a partial functional digraph with the vertex set $V(\Gamma)=$ $\left\{v_{1}, \ldots, v_{n}\right\}$. Consider the star with $n+1$ vertices $X \simeq K_{1, n}$, where $V(X)=$ $\{0, \ldots, n\}, E(X)=\{0 i: 0 \leq i \leq n\}$ and the map $\sigma: V(X) \rightarrow V(X)$ defined as follows

$$
\sigma(i)=\left\{\begin{array}{l}
j, \text { if } N_{\Gamma}^{+}\left(v_{i}\right)=\left\{v_{j}\right\}, \\
0, \text { if } i=0 \text { or } N_{\Gamma}^{+}\left(v_{i}\right)=\emptyset
\end{array}\right.
$$

for all $0 \leq i \leq n$. Then $\Gamma \simeq \Gamma(X, \sigma)$.

In particular, from Proposition 6 it follows that each disjoint union of cycles is an M-graph. In [4] it was proved that the Markov graph $\Gamma(X, \sigma)$ is a disjoint union of cycles if and only if $\sigma$ is an automorphism of $X$.

Denote by $L E(X)$ the set of leaf edges in a tree $X$. Note that the proof of Proposition 6 implies that for every partial functional digraph $\Gamma$ there exists a pair $(X, \sigma)$ such that $\Gamma \simeq \Gamma(X, \sigma)[L E(X)]$ (here $\Gamma(X, \sigma)[L E(X)]$ denotes the subgraph of $\Gamma(X, \sigma)$ induced by $L E(X))$. We can generalize this result in the following way.

Proposition 7. For a digraph $\Gamma$ there exists a pair $(X, \sigma)$ such that $\Gamma \simeq$ $\Gamma(X, \sigma)[L E(X)]$ if and only if $d_{\Gamma}^{+}(v) \leq 2$ for all $v \in V(\Gamma)$.

Proof. Clearly, for all trees $X$ and their vertex maps $\sigma$ the inequality $d_{\Gamma(X, \sigma)}^{+}(e) \geq$ 3 implies the existence of an inner edge $e^{\prime} \in N_{\Gamma(X, \sigma)}^{+}(e)$. Thus, let $\Gamma$ be a digraph with $d_{\Gamma}^{+}(v) \leq 2$ for all $v \in V(\Gamma)$ and the vertex set $V(\Gamma)=\left\{v_{1}, \ldots, v_{n}\right\}$. Consider the graph $X$ with $V(X)=\{1, \ldots, n\} \cup\left\{1^{\prime}, \ldots, n^{\prime}\right\}$ and $E(X)=\left\{i i^{\prime}\right.$ : $1 \leq i \leq n\} \cup\left\{i^{\prime} j^{\prime}: j=i+1,1 \leq i \leq n-1\right\}$. It is easy to see that $X$ is a tree with $L E(X)=\left\{i i^{\prime}: 1 \leq i \leq n\right\}$. Put

$$
\sigma(i)=\left\{\begin{array}{l}
i, \text { if } N_{\Gamma}^{+}\left(v_{i}\right)=\emptyset, \\
\max \left\{k: v_{k} \in N_{\Gamma}^{+}\left(v_{i}\right)\right\}, \text { otherwise }
\end{array}\right.
$$

and

$$
\sigma\left(i^{\prime}\right)=\left\{\begin{array}{l}
i, \text { if } N_{\Gamma}^{+}\left(v_{i}\right)=\emptyset, \\
j^{\prime}, \text { if } N_{\Gamma}^{+}\left(v_{i}\right)=\left\{v_{j}\right\}, \\
\min \left\{k: v_{k} \in N_{\Gamma}^{+}\left(v_{i}\right)\right\}, \text { if } d_{\Gamma}^{+}\left(v_{i}\right) \geq 2
\end{array}\right.
$$

for every $1 \leq i \leq n$. Then $\Gamma \simeq \Gamma(X, \sigma)[L E(X)]$. 
Proposition 8. Let $\Gamma$ be a partial functional digraph. Then $\Gamma \rightarrow K_{m}$ and $\Gamma \rightarrow \bar{K}_{m}$ are $M$-graphs for every $m \geq 1$.

Proof. Let $\Gamma$ be partial functional digraph with the vertex set $V(\Gamma)=\left\{v_{1}, \ldots, v_{n}\right\}$.

Consider the tree $X$ with $V(X)=\{0, \ldots, n\} \cup\left\{1^{\prime}, \ldots, m^{\prime}\right\}$ and $E(X)=\{0 i$ :

$1 \leq i \leq n\} \cup\left\{01^{\prime}\right\} \cup\left\{i^{\prime} j^{\prime}: j=i+1,1 \leq i \leq m-1\right\}$. Put

$$
\sigma_{1}(i)=\left\{\begin{array}{l}
j, \text { if } N_{\Gamma}^{+}\left(v_{i}\right)=\left\{v_{j}\right\}, \\
m^{\prime}, \text { if } i=0 \text { or } N_{\Gamma}^{+}\left(v_{i}\right)=\emptyset
\end{array}\right.
$$

for all $0 \leq i \leq n$ and

$$
\sigma_{1}\left(k^{\prime}\right)=\left\{\begin{array}{l}
m^{\prime}, \text { if } k \text { is even, } \\
0, \text { if } k \text { is odd }
\end{array}\right.
$$

for all $1 \leq k \leq m$. Similarly, put

$$
\sigma_{2}(i)=\left\{\begin{array}{l}
j, \text { if } N_{\Gamma}^{+}\left(v_{i}\right)=\left\{v_{j}\right\}, \\
m^{\prime}, \text { if } i=0 \text { or } N_{\Gamma}^{+}\left(v_{i}\right)=\emptyset
\end{array}\right.
$$

for every $0 \leq i \leq n$ and $\sigma_{2}\left(k^{\prime}\right)=m^{\prime}$ for $1 \leq k \leq m$. We have $\Gamma \rightarrow K_{m} \simeq$ $\Gamma\left(X, \sigma_{1}\right)$ and $\Gamma \rightarrow \bar{K}_{m} \simeq \Gamma\left(X, \sigma_{2}\right)$.

Corollary 3. For all $n, m \geq 1$ the digraphs $\bar{K}_{n} \rightarrow K_{m}$ and $\bar{K}_{n} \rightarrow \bar{K}_{m}$ are M-graphs.

Proposition 9. For all $n, m \geq 1$ the digraphs $K_{n} \rightarrow K_{m}$ and $K_{n} \rightarrow \bar{K}_{m}$ are M-graphs.

Proof. Consider the path $X \simeq P_{N}, N=n+m+1$ with $V(X)=\{1, \ldots, N\}$ and $E(X)=\{i j: j=i+1,1 \leq i, j \leq N\}$. Construct a map $\sigma_{1}: V(X) \rightarrow V(X)$ in the following way:

$$
\sigma_{1}(i)=\left\{\begin{array}{l}
N, \text { if } n+1-i \text { is even } \\
1, \text { if } 1 \leq i \leq n+1 \text { and } n+1-i \text { is odd } \\
n+1, \text { otherwise }
\end{array}\right.
$$

for all $1 \leq i \leq N$. Similarly, put

$$
\sigma_{2}(i)=\left\{\begin{array}{l}
N, \text { if } n+2 \leq i \leq N, \text { or } 1 \leq i \leq n+1 \text { and } n+1-i \text { is even, } \\
1, \text { otherwise }
\end{array}\right.
$$

for all $1 \leq i \leq N$. Then $K_{n} \rightarrow K_{m} \simeq \Gamma\left(X, \sigma_{1}\right)$ and $K_{n} \rightarrow \bar{K}_{m} \simeq \Gamma\left(X, \sigma_{2}\right)$.

It is obvious that a complete digraph $K_{n}, n \geq 1$ is realizable only on a path $P_{n+1}$. Similarly, in [4] it was proved that for $n, m \geq 1$ the complete bipartite digraph $\bar{K}_{n}+\bar{K}_{m}$ is realizable only on $P_{n+m+1}$. Finally, for $n \geq 1$ the cycle $\vec{C}_{n}$ is realizable only on $K_{1, n}$ (again, see [4] for details). 
Theorem 3. Let $\Gamma_{1}$ and $\Gamma_{2}$ be two nontrivial digraphs such that their symmetric sum $\Gamma=\Gamma_{1}+\Gamma_{2}$ is an $M$-graph. Then $\Gamma$ is realizable only on a spider of degree at most three.

Proof. Fix an isomorphism $\Gamma \simeq \Gamma(X, \sigma)$. Let the edge sets $E_{1}, E_{2} \subset E(X)$ correspond to $\Gamma_{1}$ and $\Gamma_{2}$, respectively. Obviously, $E(X)=E_{1} \sqcup E_{2}$.

At first, suppose that there exists a vertex $u \in V(X)$ with $d_{X}(u) \geq 4$. Choose four distinct vertices $u_{1}, u_{2}, u_{3}, u_{4} \in N_{X}(u)$. Without loss of generality, we can assume that $u u_{1}, u u_{2} \in E_{1}$ and $u u_{3}, u u_{4} \in E_{2}$. It is easy to see that in this case $\sigma(u) \neq u$. Again, without loss of generality, let $\sigma(u) \in A_{X}\left(u_{1}, u\right)$. Then the edge $u u_{1}$ cannot $\sigma$-cover simultaneously $u u_{3}$ and $u u_{4}$ which leads to the contradiction.

Further, suppose that there exist two vertices $u, v \in V(X)$ with $d_{X}(u)=$ $d_{X}(v)=3$. Choose two distinct vertices $u_{1}, u_{2} \in N_{X}(u)-[u, v]_{X}$ and another two distinct vertices $v_{1}, v_{2} \in N_{X}(v)-[u, v]_{X}$. Since $u \neq v$, the interval $[u, v]_{X}$ contains at least one edge. Fix such an edge $e \in E\left([u, v]_{X}\right)$. Without loss of generality, assume that $u u_{1}, v v_{1}, e \in E_{1}$ and $u u_{2}, v v_{2} \in E_{2}$. Suppose that $\sigma(u) \in A_{X}\left(u_{2}, u\right)$ and $\sigma\left(u_{1}\right) \in A_{X}\left(v_{2}, v\right)$. Then $u u_{2}$ cannot $\sigma$-cover simultaneously $u u_{1}$ and $v v_{1}$. If $\sigma(u) \in A_{X}\left(v_{2}, v\right)$ and $\sigma\left(u_{1}\right) \in A_{X}\left(u_{2}, u\right)$, then we obtain a similar contradiction. Therefore, $X$ is a spider of degree at most three.

Furthermore, let $X \simeq P_{n}, n \geq 3$ be a path with $V(X)=\{1, \ldots, n\}$ and $E(X)=\{i j: j=i+1,1 \leq i, j \leq n\}$. Consider the map

$$
\sigma(i)=\left\{\begin{array}{l}
n, \text { if } i=1 \\
2, \text { if } 1 \leq i \leq n \text { is even, } \\
1, \text { if } 3 \leq i \leq n \text { is odd }
\end{array}\right.
$$

for $1 \leq i \leq n$. Then $\Gamma(X, \sigma) \simeq \bar{K}_{1}+\bar{K}_{n-2}$ is the sum of two nontrivial digraphs.

Now suppose that $X$ is a spider of degree three. Let $u \in V(X)$ be the center of $X$ and $L(X)=\left\{v_{1}, v_{2}, v_{3}\right\}$. Therefore, $V(X)=\left[u, v_{1}\right]_{X} \cup\left[u, v_{2}\right]_{X} \cup$ $\left[u, v_{3}\right]_{X}$. Also, let $x_{i j}$ be the unique vertex from $\left[u, v_{i}\right]_{X}$ with $d_{X}\left(u, x_{i j}\right)=j$ for $1 \leq i \leq 3$ and $1 \leq j \leq d_{X}\left(u, v_{i}\right)$ (for example, $x_{i d_{X}\left(u, v_{i}\right)}=v_{i}$ for all $1 \leq i \leq 3)$. Put $\sigma(i)=\left\{\begin{array}{l}v_{1}, \text { if } x=x_{i j}, 2 \leq j \leq 3 \text { and } 1 \leq j \leq d_{X}\left(u, v_{i}\right) \text { is odd } \\ v_{2}, \text { if } x=u, \text { or } x=x_{i j}, 1 \leq i \leq 3 \text { and } 1 \leq j \leq d_{X}\left(u, v_{i}\right) \text { is even, } \\ v_{3}, \text { if } x=x_{1 j} \text { and } 1 \leq j \leq d_{X}\left(u, v_{1}\right) \text { is odd }\end{array}\right.$

for all $x \in V(X)$. Then $\Gamma(X, \sigma) \simeq \bar{K}_{d_{X}\left(u, v_{1}\right)}+\left(\bar{K}_{d_{X}\left(u, v_{3}\right)} \rightarrow K_{d_{X}\left(u, v_{2}\right)}\right)$ is the sum of two nontrivial digraphs again. Therefore, Theorem 3 provides a criterion for spiders of degree at most three. 


\section{Tournaments as M-GraphS}

In this section we present a complete list of tournaments which are Mgraphs (the so-called $t M$-graphs). Namely, consider the digraphs depicted on Figure 3.
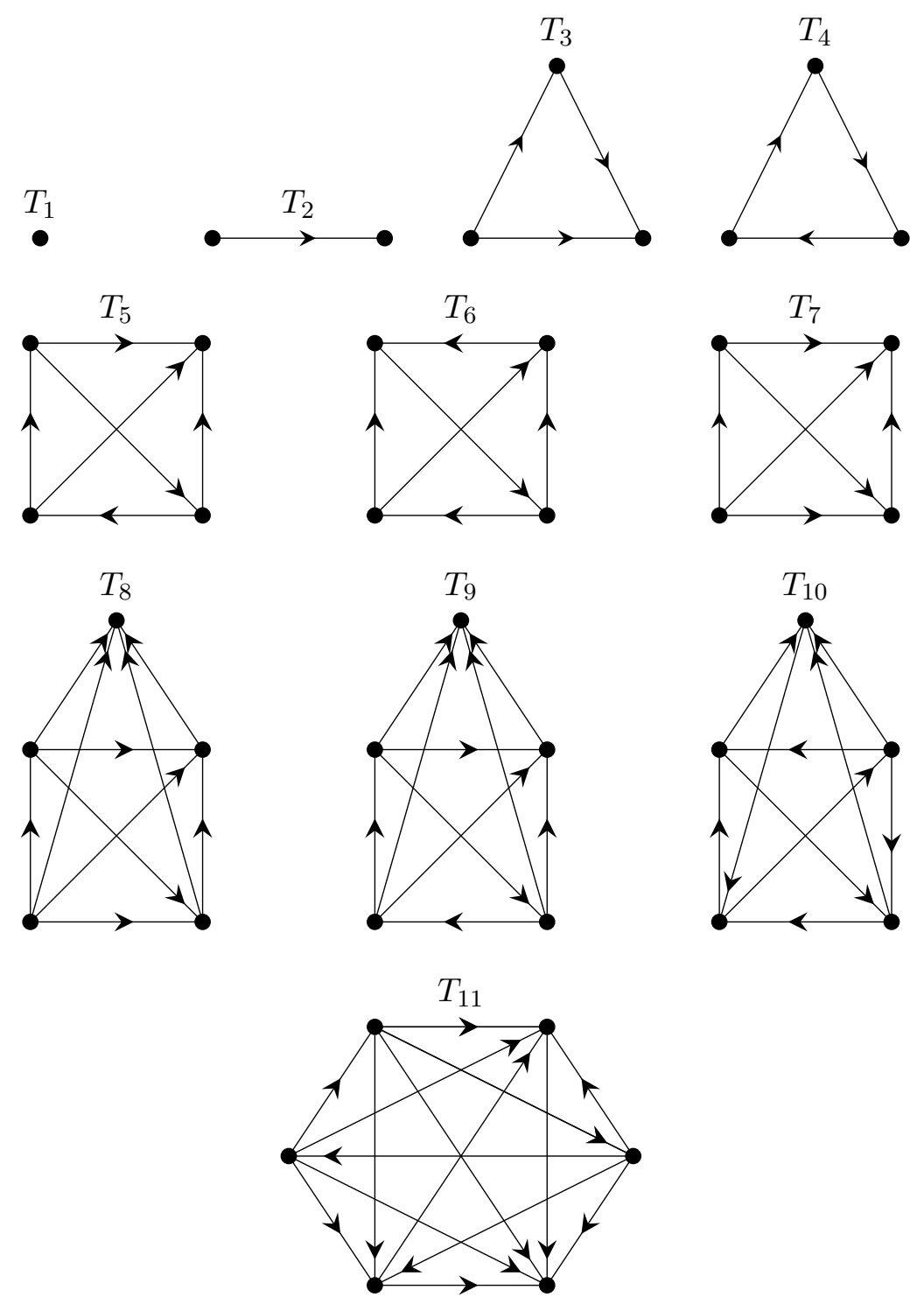

Figure 3. Eleven tM-graphs.

It is easy to see (for example, by checking indegree sequences) that all digraphs $T_{i}, 1 \leq i \leq 11$ are pairwise non-isomorphic tournaments.

Lemma 2. The tournaments $T_{i}, 1 \leq i \leq 11$ are M-graphs. 
Proof. Consider the next trees $X_{i}$ and their maps $\sigma_{i}: V\left(X_{i}\right) \rightarrow V\left(X_{i}\right)$ for $1 \leq i \leq 11$ :

$$
\begin{aligned}
& V\left(X_{1}\right)=\{1,2\}, E\left(X_{1}\right)=\{12\}, \sigma_{1}=\left(\begin{array}{cc}
1 & 2 \\
1 & 1
\end{array}\right) \text {. } \\
& V\left(X_{2}\right)=\{1,2,3\}, E\left(X_{2}\right)=\{12,23\}, \sigma_{2}=\left(\begin{array}{lll}
1 & 2 & 3 \\
3 & 2 & 2
\end{array}\right) . \\
& V\left(X_{3}\right)=\{1,2,3,4\}, E\left(X_{3}\right)=\{12,23,34\}, \sigma_{3}=\left(\begin{array}{llll}
1 & 2 & 3 & 4 \\
2 & 4 & 3 & 3
\end{array}\right) . \\
& V\left(X_{4}\right)=\{1,2,3,4\}, E\left(X_{4}\right)=\{12,13,14\}, \sigma_{4}=\left(\begin{array}{cccc}
1 & 2 & 3 & 4 \\
1 & 3 & 4 & 2
\end{array}\right) . \\
& V\left(X_{5}\right)=\{1, \ldots, 5\}, E\left(X_{5}\right)=\{12,13,14,15\}, \sigma_{5}=\left(\begin{array}{ccccc}
1 & 2 & 3 & 4 & 5 \\
2 & 2 & 4 & 5 & 3
\end{array}\right) . \\
& V\left(X_{6}\right)=\{1, \ldots, 5\}, E\left(X_{6}\right)=\{12,23,24,45\}, \sigma_{6}=\left(\begin{array}{ccccc}
1 & 2 & 3 & 4 & 5 \\
3 & 2 & 5 & 1 & 4
\end{array}\right) . \\
& V\left(X_{7}\right)=\{1, \ldots, 5\}, E\left(X_{7}\right)=\{12,23,34,45\}, \sigma_{7}=\left(\begin{array}{ccccc}
1 & 2 & 3 & 4 & 5 \\
2 & 5 & 3 & 3 & 4
\end{array}\right) . \\
& V\left(X_{8}\right)=\{1, \ldots, 6\}, E\left(X_{8}\right)=\{12,23,34,45,46\}, \sigma_{8}=\left(\begin{array}{cccccc}
1 & 2 & 3 & 4 & 5 & 6 \\
2 & 3 & 3 & 1 & 6 & 4
\end{array}\right) . \\
& V\left(X_{9}\right)=\{1, \ldots, 6\}, E\left(X_{9}\right)=\{12,23,25,26,34\}, \sigma_{9}=\left(\begin{array}{cccccc}
1 & 2 & 3 & 4 & 5 & 6 \\
5 & 4 & 3 & 3 & 6 & 1
\end{array}\right) . \\
& V\left(X_{10}\right)=\{1, \ldots, 6\}, E\left(X_{10}\right)=\{12,23,34,36,45\}, \sigma_{10}=\left(\begin{array}{cccccc}
1 & 2 & 3 & 4 & 5 & 6 \\
2 & 5 & 3 & 6 & 4 & 1
\end{array}\right) . \\
& V\left(X_{11}\right)=\{1, \ldots, 7\}, E\left(X_{11}\right)=\{12,23,26,27,34,45\}, \sigma_{11}=\left(\begin{array}{ccccccc}
1 & 2 & 3 & 4 & 5 & 6 & 7 \\
6 & 5 & 3 & 3 & 4 & 7 & 1
\end{array}\right) .
\end{aligned}
$$

Direct computations show that $T_{i} \simeq \Gamma\left(X_{i}, \sigma_{i}\right)$ for all $1 \leq i \leq 11$.

Thus, we want to prove that there is no other tM-graphs except of $T_{i}$, $1 \leq i \leq 11$.

Lemma 3. Let $X$ be a tree, $\sigma: V(X) \rightarrow V(X)$ be some map and $\Gamma=\Gamma(X, \sigma)$ be a tournament. Then $\operatorname{diam} X \leq 4$.

Proof. Since $\Gamma$ does not have vertices with loops, $\sigma$ is an anti-expansive map. Therefore, from Proposition 2 it follows that there exists $u_{0} \in V(X)$ such that $u \rightarrow v$ in $X\left(\tau_{\sigma}\right)$ implies $v \in\left[u_{0}, u\right]_{X}$.

Suppose that $\operatorname{ecc} X\left(u_{0}\right) \geq 3$. Choose three distinct vertices $u_{1}, u_{2}, u_{3} \in$ $V(X)$ such that $\left[u_{0}, u_{3}\right]_{X}=\left\{u_{0}-u_{1}-u_{2}-u_{3}\right\}$. Since $u_{0} u_{1} \nrightarrow u_{1} u_{2}$ in $\Gamma$, we have $u_{1} u_{2} \rightarrow u_{0} u_{1}$ in $\Gamma$. Therefore, $\sigma\left(u_{2}\right) \in A_{X}\left(u_{1}, u_{0}\right)$. Similarly, $u_{0} u_{1} \nrightarrow$ $u_{2} u_{3}$ in $\Gamma$ means that $u_{2} u_{3} \rightarrow u_{0} u_{1}$ in $\Gamma$ and thus $\sigma\left(u_{3}\right) \in A_{X}\left(u_{0}, u_{1}\right)$. Now it is easy to see that $u_{1} u_{2} \nrightarrow u_{2} u_{3}$ and $u_{2} u_{3} \nrightarrow u_{1} u_{2}$ in $\Gamma$. But this is impossible since $\Gamma$ is a tournament. Thus, $\operatorname{diam} X \leq 2 \cdot \operatorname{rad} X \leq 2 \cdot \operatorname{ecc}_{X}\left(u_{0}\right) \leq 4$.

Corollary 4. Let $X$ be a tree with $n \geq 1$ vertices, $\sigma: V(X) \rightarrow V(X)$ be some map and $\Gamma=\Gamma(X, \sigma)$ be a tournament. Then $n \leq 10$. 
Proof. Since $\Gamma$ is a tournament, $|A(\Gamma)|=\sum_{e \in E(X)} d_{\Gamma}^{+}(e)=\frac{(n-1)(n-2)}{2}$. This means that there exists an edge $e \in E(X)$ such that

$$
d_{\Gamma}^{+}(e) \geq \frac{(n-1)(n-2)}{2} \cdot \frac{1}{n-1}=\frac{n-2}{2} .
$$

Combining this with Proposition 1 and Lemma 3, we obtain

$$
\frac{n-2}{2} \leq d_{\Gamma}^{+}(e) \leq \operatorname{diam} X \leq 4
$$

which yields $n \leq 10$.

Thus, in order to find all tM-graphs, one must check all Markov graphs $\Gamma(X, \sigma)$ for (finite number) of trees $X$ with $\operatorname{diam} X \leq 4$ and $|V(X)| \leq 10$. Of course, this can be done by direct "brute-force" computations. However, one can simplify the task by considering forbidden mixed subtrees for $X\left(\tau_{\sigma}\right)$. Namely, if $\Gamma$ is a nontrivial tM-graph, then there exists a vertex $v \in V(\Gamma)$ such that $\Gamma-\{v\}$ is also a tM-graph.

Now we sketch the strategy of such direct computations. Checking pairs $(X, \sigma)$ for trees $X$ with $|V(X)| \leq 3$ it is easy to observe that the only tMgraphs with at most two vertices are $T_{1}$ and $T_{2}$. From Lemma 3 and Proposition 2 it follows that the directed path with four vertices $\Gamma_{1}=\{\bullet \rightarrow \bullet \rightarrow \bullet \rightarrow$ - $\}$ is the forbidden subtree for each $X\left(\tau_{\sigma}\right)$ for all pairs $(X, \sigma)$ with $\Gamma(X, \sigma)$ being a tournament. Another admissible (in the sense of Proposition 2) oriented trees with four vertices are realizable as mixed trees $X\left(\tau_{\sigma}\right)$ for pairs $(X, \sigma)$ with $\Gamma(X, \sigma)$ being a tournament for some proper maps $\sigma$. Therefore, we must consider pairs $(X, \sigma)$ for trees $X$ with $|V(X)|=5$. Figure 4 depicts the five possibilities for $X\left(\tau_{\sigma}\right)$.

It is easy to observe that $\Gamma_{2}$ is another forbidden subtree, while the other four oriented trees produce Markov graphs isomorphic to $T_{5}, T_{6}$ and $T_{7}$ (for suitable maps). Also, observe that since $\Gamma_{2}$ is a forbidden subtree, for every tournament $\Gamma(X, \sigma)$, where $|V(X)| \geq 6$ it holds $\Delta(X) \leq 4$. Thus, we obtain the next four possibilities for $X\left(\tau_{\sigma}\right)$ for pairs $(X, \sigma)$ with $\Gamma(X, \sigma)$ being a tournament, where $|V(X)|=6$ (see Figure 5 ).

Again, some computations involving enumeration of all maps $\sigma$ show that $\Gamma_{3}$ is a forbidden subtree and the other three oriented trees produce Markov graphs isomorphic to $T_{8}, T_{9}$ and $T_{10}$. Further, having in mind the forbidden subtrees $\Gamma_{1}, \Gamma_{2}$ and $\Gamma_{3}$ consider the next two possibilities for $X\left(\tau_{\sigma}\right)$ for pairs $(X, \sigma)$ with $\Gamma(X, \sigma)$ being a tournament, where $|V(X)|=7$ (see Figure 6).

One can show that $\Gamma_{4}$ is another forbidden subtree, while the other tree produces a Markov graph isomorphic to $T_{11}$. Finally, for trees $X$ with $|V(X)| \geq 8$ and their anti-expansive maps $\sigma$ each mixed tree $X\left(\tau_{\sigma}\right)$ contains a subtree isomorphic to either $\Gamma_{1}, \Gamma_{2}, \Gamma_{3}$ or $\Gamma_{4}$. Thus, we have the following result. 


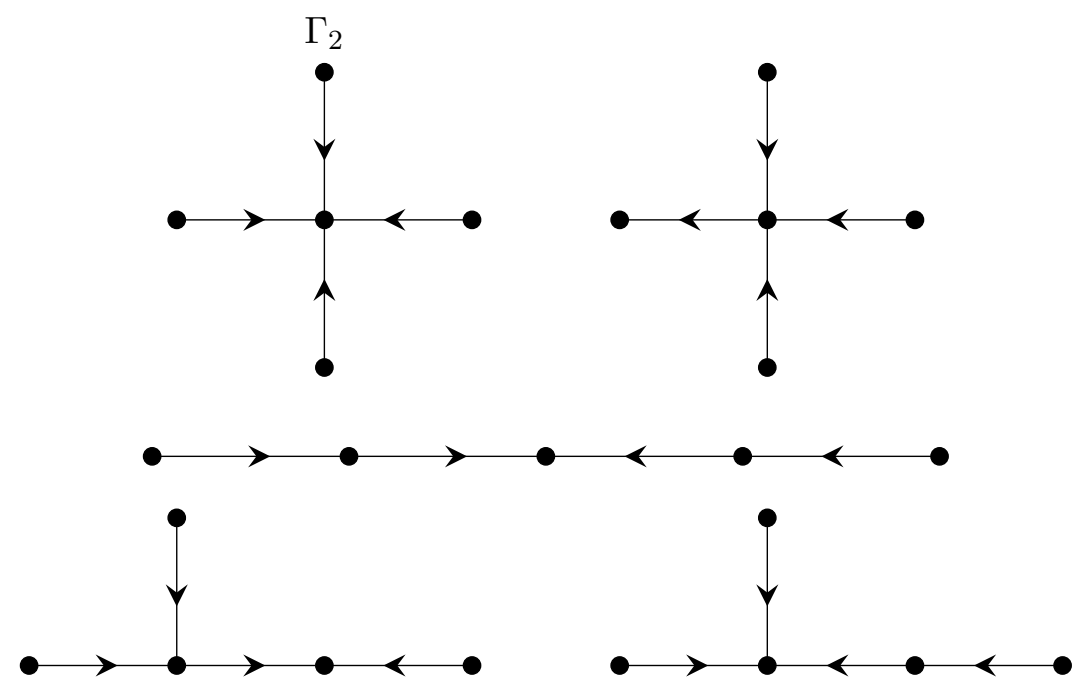

Figure 4. Mixed trees $X\left(\tau_{\sigma}\right)$ for $X$ with $|V(X)|=5$ and anti-expansive maps $\sigma$, which do not contain $\Gamma_{1}$ as a subtree.
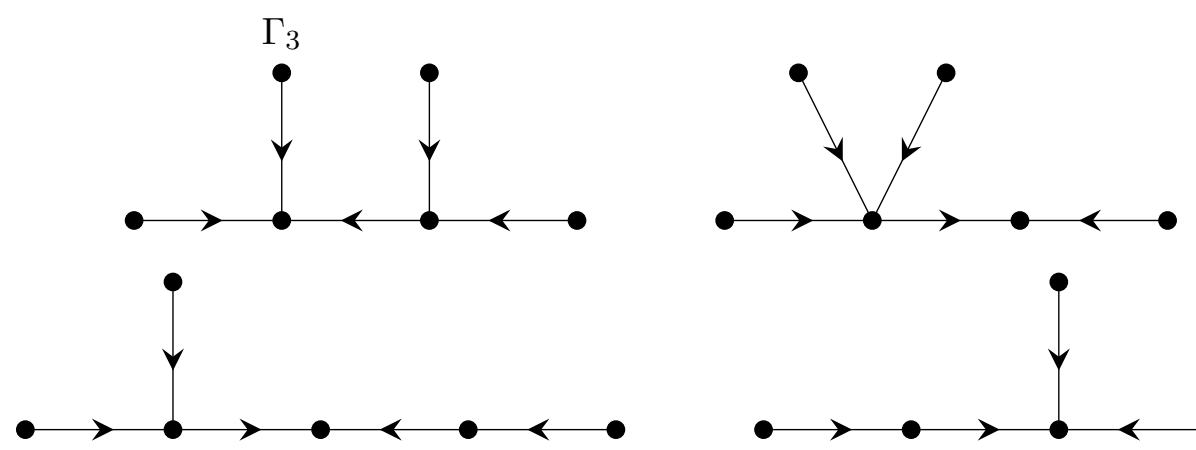

Figure 5. Mixed trees $X\left(\tau_{\sigma}\right)$ for $X$ with $|V(X)|=6$ and antiexpansive maps $\sigma$, which do not contain $\Gamma_{1}, \Gamma_{2}$ as subtrees.
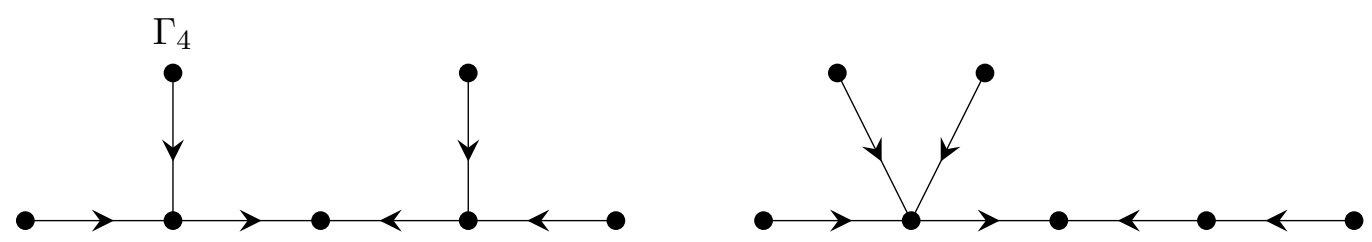

Figure 6. Mixed trees $X\left(\tau_{\sigma}\right)$ for $X$ with $|V(X)|=7$ and antiexpansive maps $\sigma$, which do not contain $\Gamma_{1}, \Gamma_{2}, \Gamma_{3}$ as subtrees.

Theorem 4. Let $\Gamma$ be a nontrivial M-graph. Then $\Gamma$ is a tournament if and only if $\Gamma \simeq T_{i}$ for some $1 \leq i \leq 11$. 


\section{M-GRAPHS With THREE VERTICES}

It is easy to see that each digraph with at most two vertices is an M-graph. In this section we provide a complete list of 3-vertex M-graphs. Recall that there is only two non-isomorphic 4-vertex trees: $P_{4}$ and $K_{1,3}$. Checking all possible maps on these two trees, we can conclude that there exist exactly 86 pairwise non-isomorphic 3-vertex M-graphs. Note that the number of pairwise non-isomorphic digraphs with three vertices equals 104 (see [10]). Therefore, there is 18 pairwise non-isomorphic 3-vertex digraphs which are not M-graphs (see Figure 7).

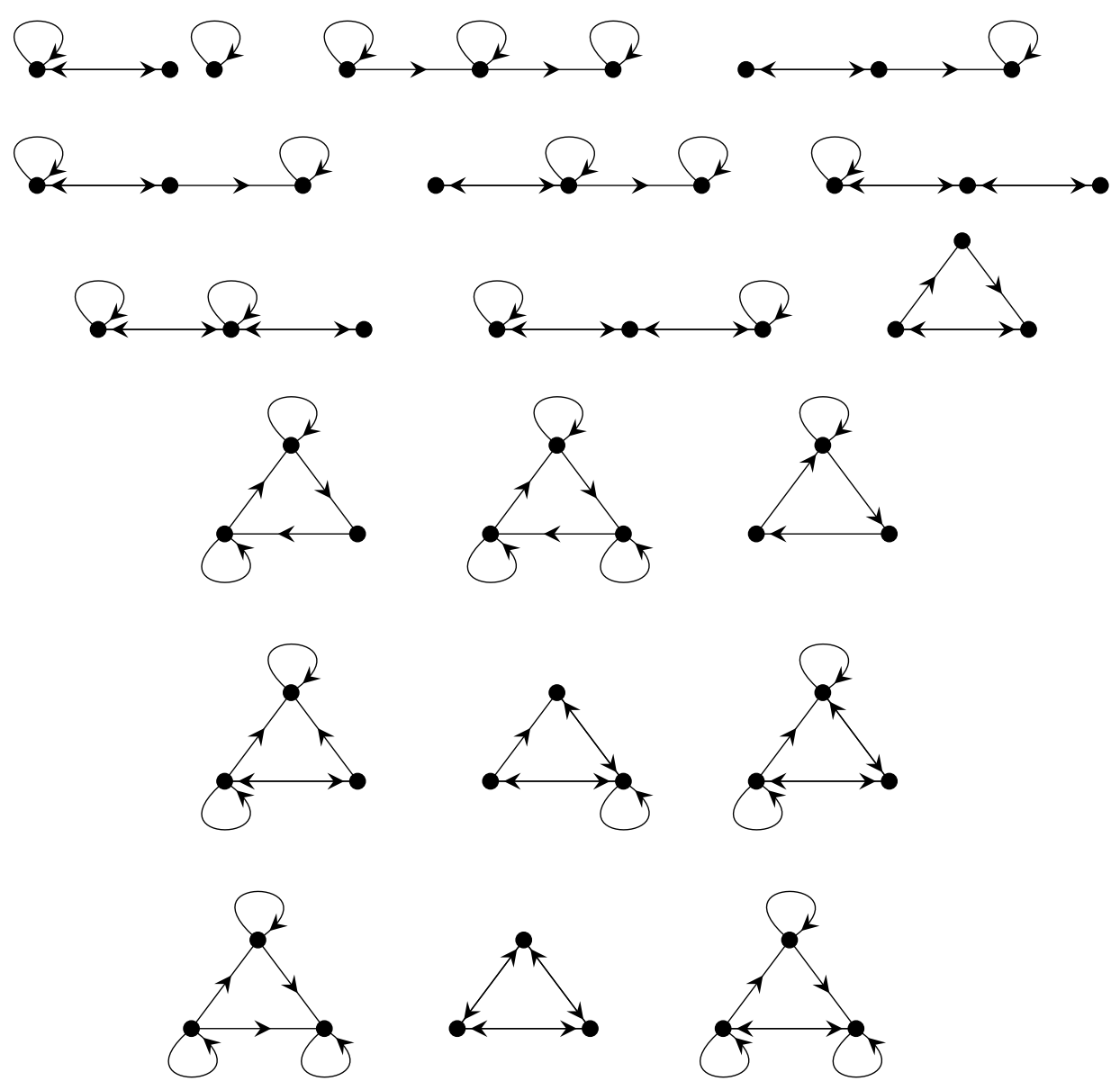

Figure 7. Complete list of 3-vertex digraphs which are not M-graphs.

\section{REFERENCES}

[1] C. Bernhardt, Vertex maps for trees: algebra and periods of periodic orbits, Disc. Contin. Dyn. Syst., 14 (2006), 399-408. 
[2] C-W. Ho and C. Morris, A graph theoretic proof of Sharkovsky's theorem on the periodic points of continuous functions, Pacific J. Math., 96 (1981), 361-370.

[3] S. Kozerenko, On Markov graphs, Algebra Discrete Math., 16 (2013), 96-102.

[4] S. Kozerenko, Markov graphs of one-dimensional dynamical systems and their discrete analogues, Rom. J. Math. Comput. Sci., 6 (2016), 16-24.

[5] S. Kozerenko, Discrete Markov graphs: loops, fixed points and maps preordering, $J$. Adv. Math. Stud., 9 (2016), 99-109.

[6] V. A. Pavlenko, Number of digraphs of periodic points of a continuous mapping of an interval into itself, Ukrainian Math. J., 39 (1987), 481-486.

[7] V. A. Pavlenko, Periodic digraphs and their properties, Ukrainian Math. J., 40 (1988), 455-458.

[8] V. A. Pavlenko, On characterization of periodic digraphs, Cybernet. Systems Anal., 25 (1989), 49-54.

[9] A. N. Sharkovsky, Coexistence of cycles of continuous mapping of the line into itself, Ukrainian Math. J., 16 (1964), 61-71.

[10] N. J. A. Sloane, The On-Line Encyclopedia of Integer Sequences, http://oeis.org. Sequence A000595.

[11] P. D. Straffin, Periodic points of continuous functions, Math. Mag., 51 (1978), 99-105.

Faculty of Mechanics and Mathematics, Taras Shevchenko National UniVERSITY OF KYIV, VOLODYMYRSKA STR. 64, 01033 KYIV, UKRAINE

E-mail address: kozerenkosergiy@ukr.net 